\title{
Analysis of JDAM Tests at China Lake
}

\author{
Kenneth Welling \\ Department of Electrical \& Computer Engineering \\ Brigham Young University \\ Provo, UT 84602 \\ wellingk@ift.ee.byu.edu
}

Faculty Advisor:

Dr. Michael Rice

\begin{abstract}
This paper examines telemetry signal levels of the Joint Direct Air Munition (JDAM) tests at China Lake NAWC. Significant received signal level variations were observed as the munition approached impact. This paper presents and examines two simple multipath models to explain the received signal variations. The first model assumes a flat earth and a single specular multipath reflection. The second model uses the actual contour of the earth and considers a number of specular multipath reflections. The first model (which assumes the single multipath reflection) more accurately reproduces the variations in the received signal level than the second.
\end{abstract}

\section{INTRODUCTION}

The Joint Direct Air Munition (JDAM) was tested at China Lake NAWC during January 1996. This testing provided a unique opportunity to study the relationship between elevation angle and multipath interference since the slant range remained nearly constant as the bomb fell. Significant variations in the received signal level were observed near the end of the test. Since these variations occured when the JDAM-to-receive-sight elevation angles were small, multipath interference is a likely cause. Previous models of multipath interference assume a flat earth with a single specular reflection $[1,2,3]$ as illustrated in Figure 1. In addition to the single multipath reflection model, this paper considers the effect of variations in the earth's surface which cause multiple multipath reflections as illustrated in Figure 2. 


\section{SIGNAL DATA COLLECTION}

Received signal levels were derived from Automatic Gain Control (AGC) voltage levels recorded from a Microdyne 1200 MR receiver during the JDAM test run released at 22:39:55.5 on January 23,1997 . The telemetry data were transmitted at $2362.5 \mathrm{MHz}$ to the 6 foot dish at the receiving station located 15.5 miles $(25 \mathrm{Km})$ from the impact point.

The Automatic Gain Control (AGC) levels were recorded on magnetic tape, then later played back and sampled at $20 \mathrm{ksamples} / \mathrm{sec}$ using a 12-bit A/D converter described in [4]. Received signal power $v_{s}$ was determined from the AGC voltage samples using a pointslope approximation obtained from a calibration procedure.

\section{MULTIPATH GEOMETRY}

The JDAM Time and Space Position Information (TSPI) were only available for the last five seconds of flight, so the release point and trajectory were calculated from the first available data using the following assumptions:

1. Initial vertical velocity of $0 \mathrm{~m} / \mathrm{s}$.

2. Initial horizontal velocity of 0.8 Mach.

3. Vertical acceleration of $9.8 \mathrm{~m} / \mathrm{s}^{2}$.

4. Horizontal acceleration of $-4.5 \mathrm{~m} / \mathrm{s}^{2}$. This value was computed as the average of the horizontal velocity at release $(0.8 \mathrm{Mach})$ and the horizontal velocity at the last available data point $(81.9 \mathrm{~m} / \mathrm{s})$ divided by the time difference between these two points (40.3 s).

Using these assumptions, the munition was estimated to have been released at an altitude of 23,425 feet $(7140 \mathrm{~m})$, and to have traversed 3.89 miles $(6.261 \mathrm{~km})$ along the ground, as illustrated in Figure 3.

Earth contour data was derived from USGS contour maps sampled every $1 / 3$ mile $(536.5 \mathrm{~m})$. The contour slice along the receive site/JDAM impact point is illustrated in Figure 3. Note the presence of the White Hills $21 \mathrm{~km}$ away from the receive site. The impact point was visually obscured from the receive site by these mountains. During the test, the munition disappeared behind the White Hills and the receive signal was lost approximately 0.5 seconds prior to impact. The White Hills also provided a source for multiple signal reflections as the line-of-sight path skirted the tops of these mountains. 


\section{MULTIPATH MODELS}

The first and simplest multipath model assumed a flat surface between the receiver and impact point, resulting in exactly one specular reflection at any given time as illustrated in Figure 1. Assuming a constant receiver height of $7.62 \mathrm{~m}$ (which is the height of the receiving station relative to the desert floor $1 / 3$ mile away), all reflections for the JDAM trajectory occured within 0.5 miles of the receiving site. A dielectric constant of ${ }_{r}=2.8-j 0.2$ was used to compute $\Gamma(\mathrm{N})$ which defines the magnitude and phase of the specular reflection as a function of angle using [5]:

$$
\Gamma(\phi)=\frac{\cos \left(90^{\circ}-\phi\right)-a \sqrt{\epsilon_{r}-\sin ^{2}\left(90^{\circ}-\phi\right)}}{\cos \left(90^{\circ}-\phi\right)+a \sqrt{\epsilon_{r}-\sin ^{2}\left(90^{\circ}-\phi\right)}}
$$

where $a={ }_{r}$ for vertical polarization or 1 for horizontal polarization. (Horizontal polarization was assumed for the simulations.) The reflection point is the point where the incidence angle (and hence the reflection angle) are such that the specular reflection arrives at the receive antenna. Since the propagation distance of the multipath reflection $\left(m_{1}+m_{2}\right)$ is slightly larger than the line-of-sight propagation distance $(r)$, the differential delay $\tau$ causes an additional phase shift in the specular reflection.

The received power levels were computed using

$$
P=P_{t} G_{t} G_{r, \max }\left(\frac{\lambda}{4 \pi}\right)^{2}\left|\frac{1}{r}+\frac{\Gamma(\phi) e^{-j 2 \pi f_{0} \tau}}{m_{1}+m_{2}} \sqrt{G_{r}\left(\theta_{\mathrm{el}}+\phi\right)}\right|^{2} .
$$

where $P_{t}$ is the transmit power, $G_{t}$ is the transmit antenna gain, $G_{r, \max }$ is the receive antenna gain at boresight, and $G_{r}\left(2_{\mathrm{el}}+\mathrm{N}\right)$ is the off-boresight receive antenna gain relative to the boresight gain which is included to account for the effects of the antenna gain pattern on reflections arriving off the main lobe [6]. Using this model, the receive signal levels were computed and compared with the measured signal levels. The comparison is shown in Figure 4 where generally close agreement between the model and the data is observed, especially during the last 2 seconds.

Differences in the two are due, in part, to the single multipath reflection assumption and phase differences between the actual reflections and those predicted using the assumed dielectric constant. In addition, the simple model predicts significant multipath interference when the reflections arrive within the main lobe of the receive antenna gain pattern (which has a beam width of $\pm 4.9^{\circ}$. That is, multipath interference is occurs only when $2_{\mathrm{el}}+\mathrm{N}<4.9^{\circ}$ which is the case during the last 3 seconds of the test. The data show 
significant multipath interference before that time, indicating that the assumed geometry is too simple to account for all the observed multipath reflections.

The second multipath model took the contour of the ground into consideration. Multiple reflections are possible since uneven surfaces between the transmitter and receiver generate several locations where the incidence/reflection angles define transmitter to receiver paths. In this case, the received power is given by

$$
P=P_{t} G_{t} G_{r, \max }\left(\frac{\lambda}{4 \pi}\right)^{2}\left|\frac{1}{r}+\sum_{n} \frac{\Gamma\left(\phi_{n}\right) e^{-j 2 \pi f_{0} \tau_{n}}}{m_{1, n}+m_{2, n}} \sqrt{G_{r}\left(\theta_{\mathrm{el}, n}+\phi_{n}\right)}\right|^{2} .
$$

where the subscript $n$ denotes the geometrical parameters of the $n$-th reflected path. The result of this model is compared to actual received signal voltage levels in Figure 5. The additional complexity associated with the multiple reflection model produces in extremely modest improvements in the model accuracy. In fact, it is not clear in this case that improvement results from the inclusion of the additional multipath reflections. This could be attributed to the coarse spatial sampling of the contours along the trajectory. Since the dielectric is not known precisely and varies over the trajectory, the predicted phase shifts associated with the reflections are not accurate. Using multiple inaccurate estimates has its limitations.

\section{EVALUATION}

This paper demonstrates that the signal variations in the JDAM test data are well modeled using a multipath assumption. Both the data and the model show multipath interference increasing with decreasing elevation angle although the data demonstrate significant multipath interference at higher elevation angles than that predicted by the models. The single multiple reflection reproduces reasonably well the observed variations. The additional complexity associated with the multiple reflection model does not seem to improve the modeling capability significantly. Ideally, one would expect the multiple reflection model to accurately reproduce the observed received signal variations if the ground contours were measured every foot and the exact dielectric constant at each contour location was known. It also seems clear that there were other factors affecting received signal levels during the test such as variations in the transmit antenna gain pattern.

\section{REFERENCES}

[1] N. Tom Nelson. Probability of bit error on a standard IRIG telemetry channel using the aeronautical fading channel model. In Proceedings of the International Telemetering Conference, volume 30, pages 356-363, October 1994. 
[2] Lyman Horne and Ricky Dye. Parameter characterization on a telemetry channel including the effects of the specular ray. In Proceedings of the International Telemetering Conference, volume 31, pages 210-215, October 1995.

[3] Michael Rice and Gene Law. Aeronautical telemetry fading sources. In Proceedings of the International Telemetering Conference, volume 33, October 1997.

[4] Lyman Horne and Ricky Dye. An inexpensive data acquisition system for measuring telemetry signals on test ranges to estimate channel characteristics. In Proceedings of the International Telemetering Conference, volume 31, pages 210-215, October 1995.

[5] Howard Xia et. al. Radio propagation characteristics for line-of-sight microcellular and personal communications. IEEE Transactions on Antennas and Proagation, 41(10):1439-1447, October 1993.

[6] Michael Rice and Daniel Friend. Antenna gain pattern effects on multipath interference in aeronautical telemetering. In Proceedings of the International Telemetering Conference, volume 33, October 1997. 


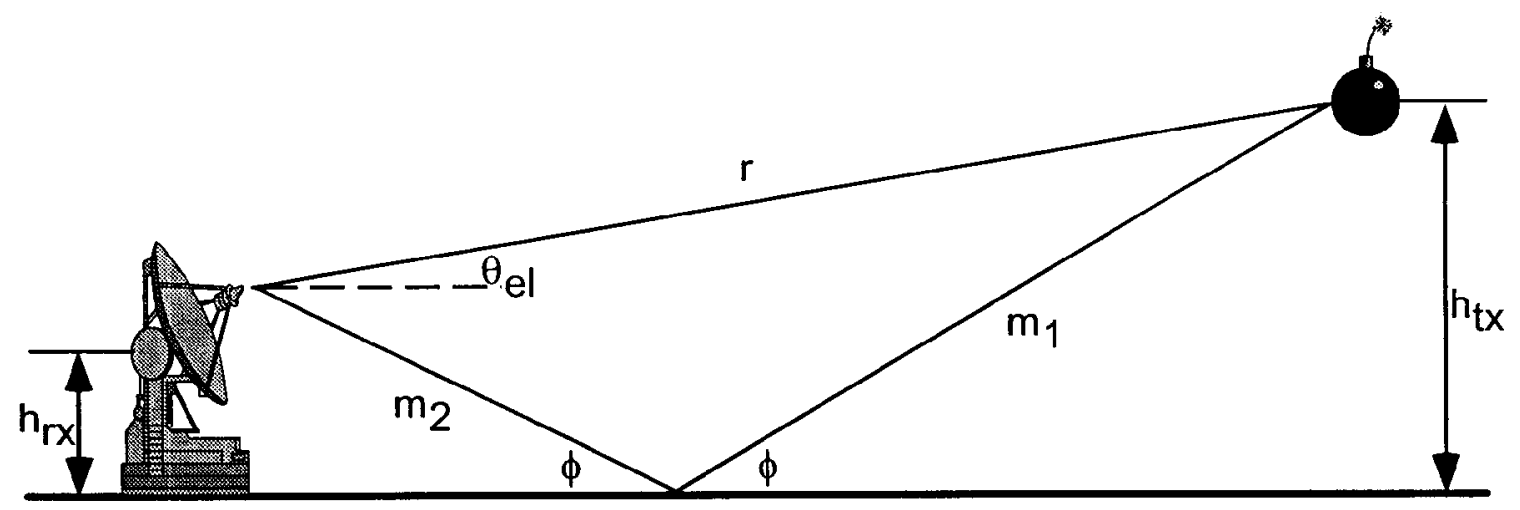

Figure 1: Simple Flat Earth Multipath Model for Aeronautical Telemetry

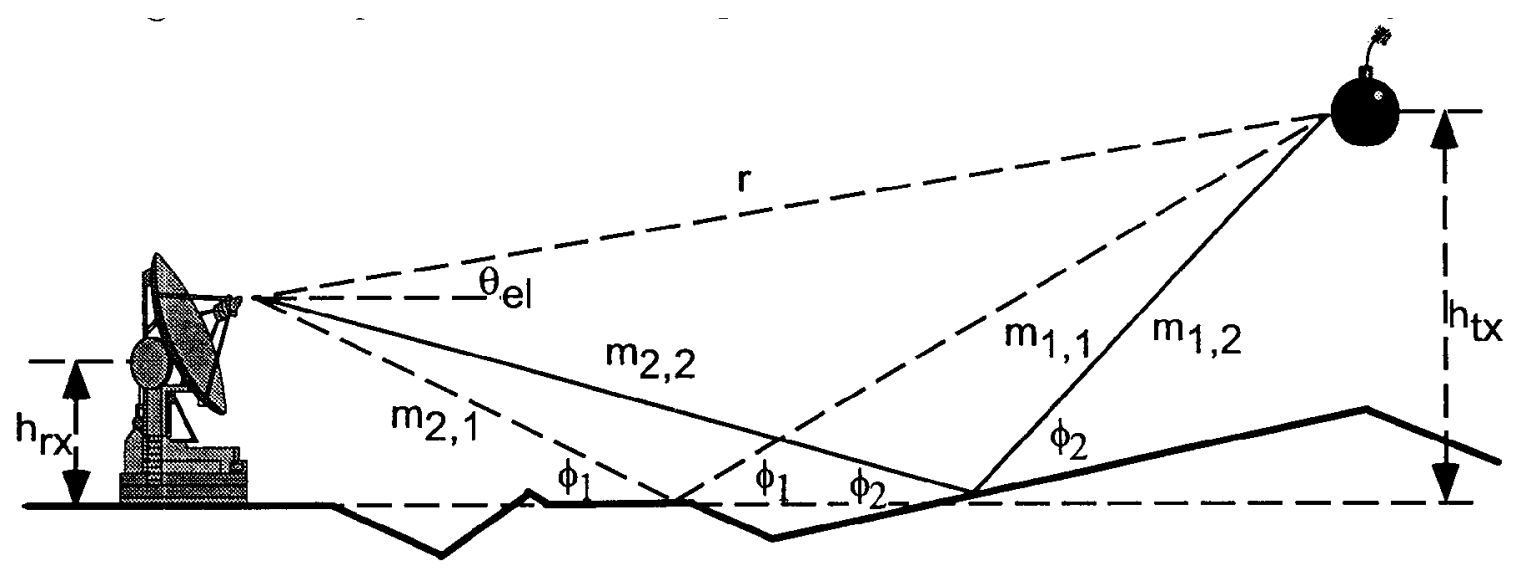

Figure 2: Contoured Earth Multipath Model for Aeronautical Telemetry With Multiple Reflections 

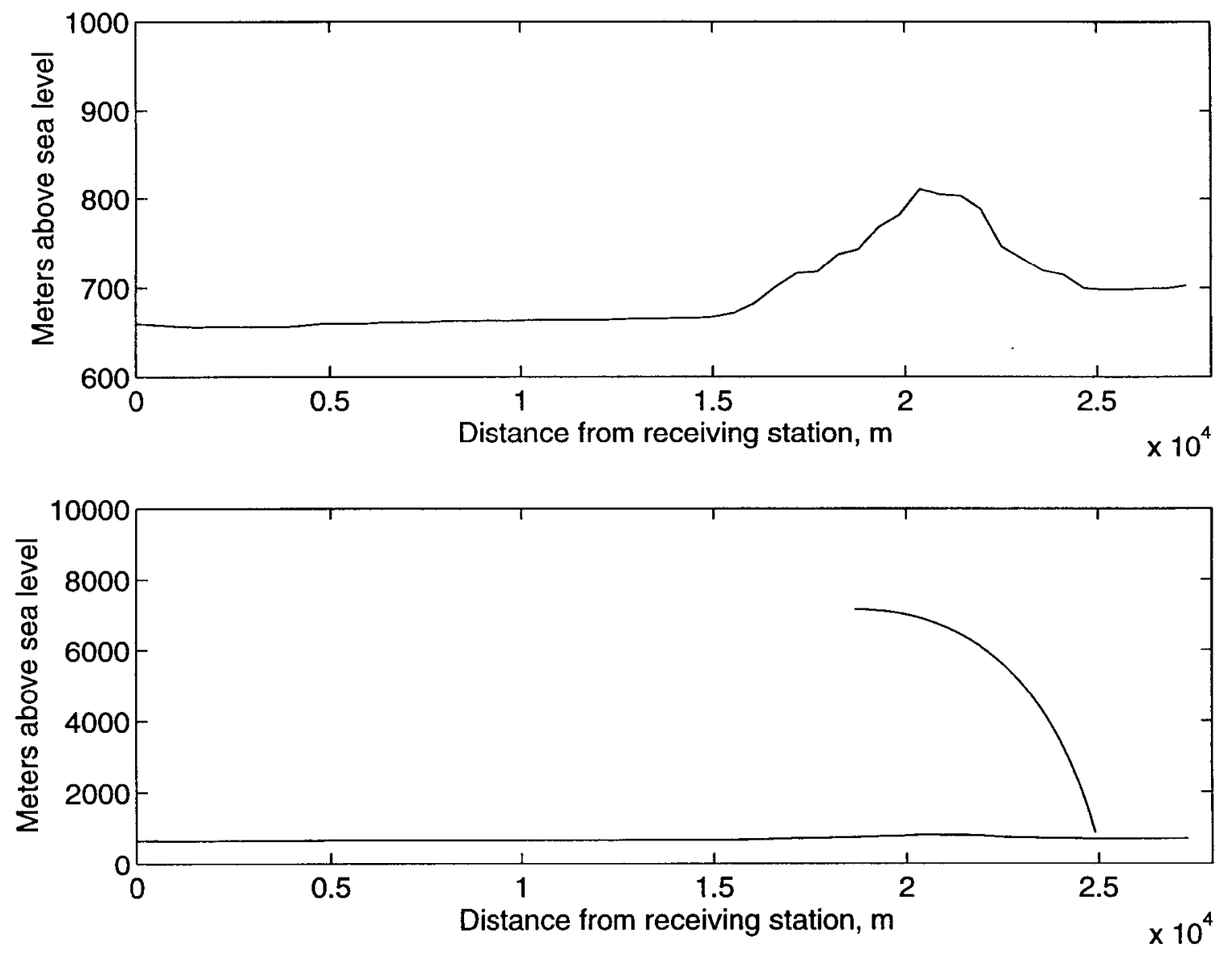

Figure 3: Contour of the land on the line directly between the receiving station $(0 \mathrm{~m})$ and the impact point $(25000 \mathrm{~m})$, shown again with the estimated JDAM trajectory. 

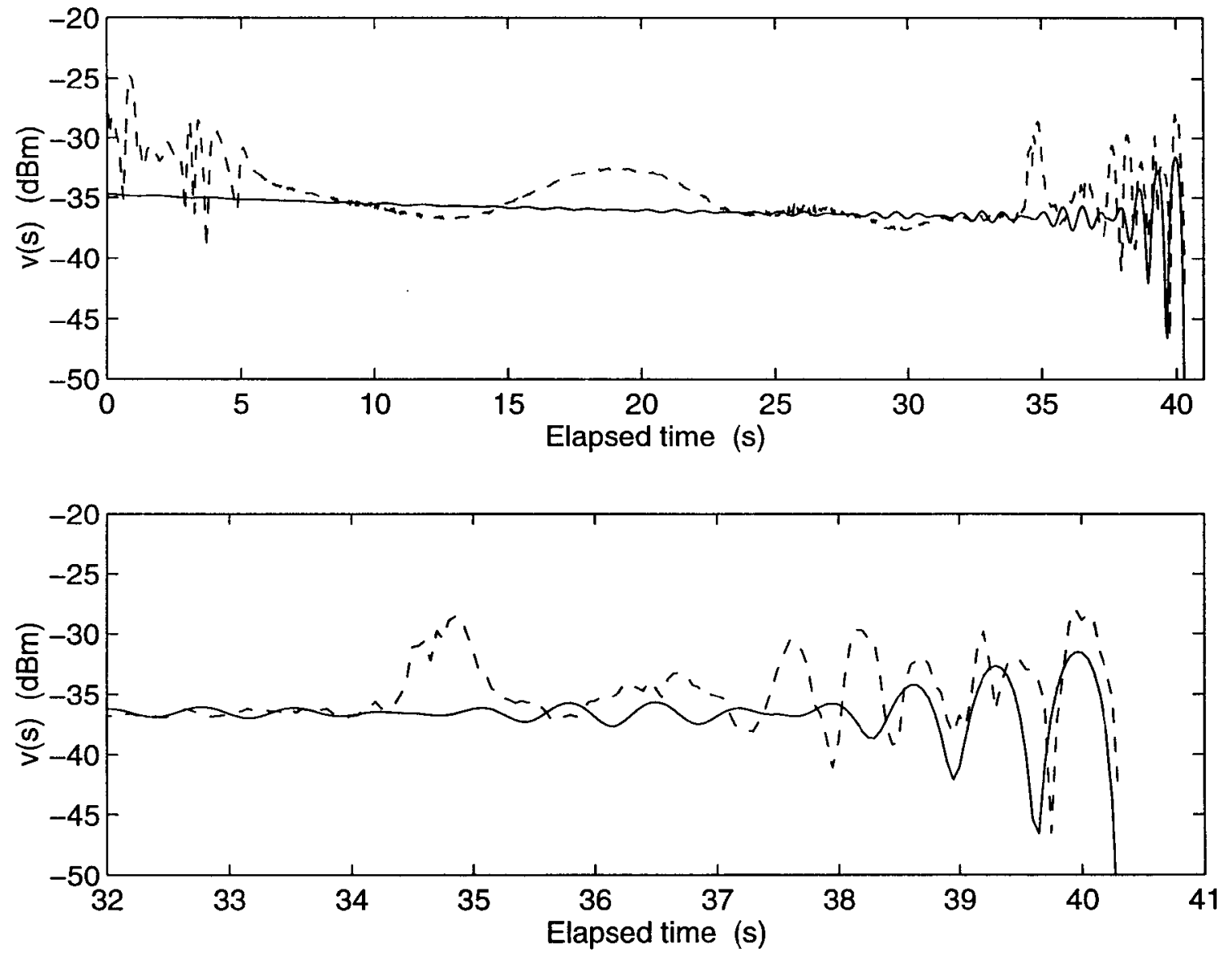

Figure 4: Signal voltage levels from actual data run (dashed line) compared to multipath model assuming a flat reflecting surface, involving a single specular path (solid-line). 

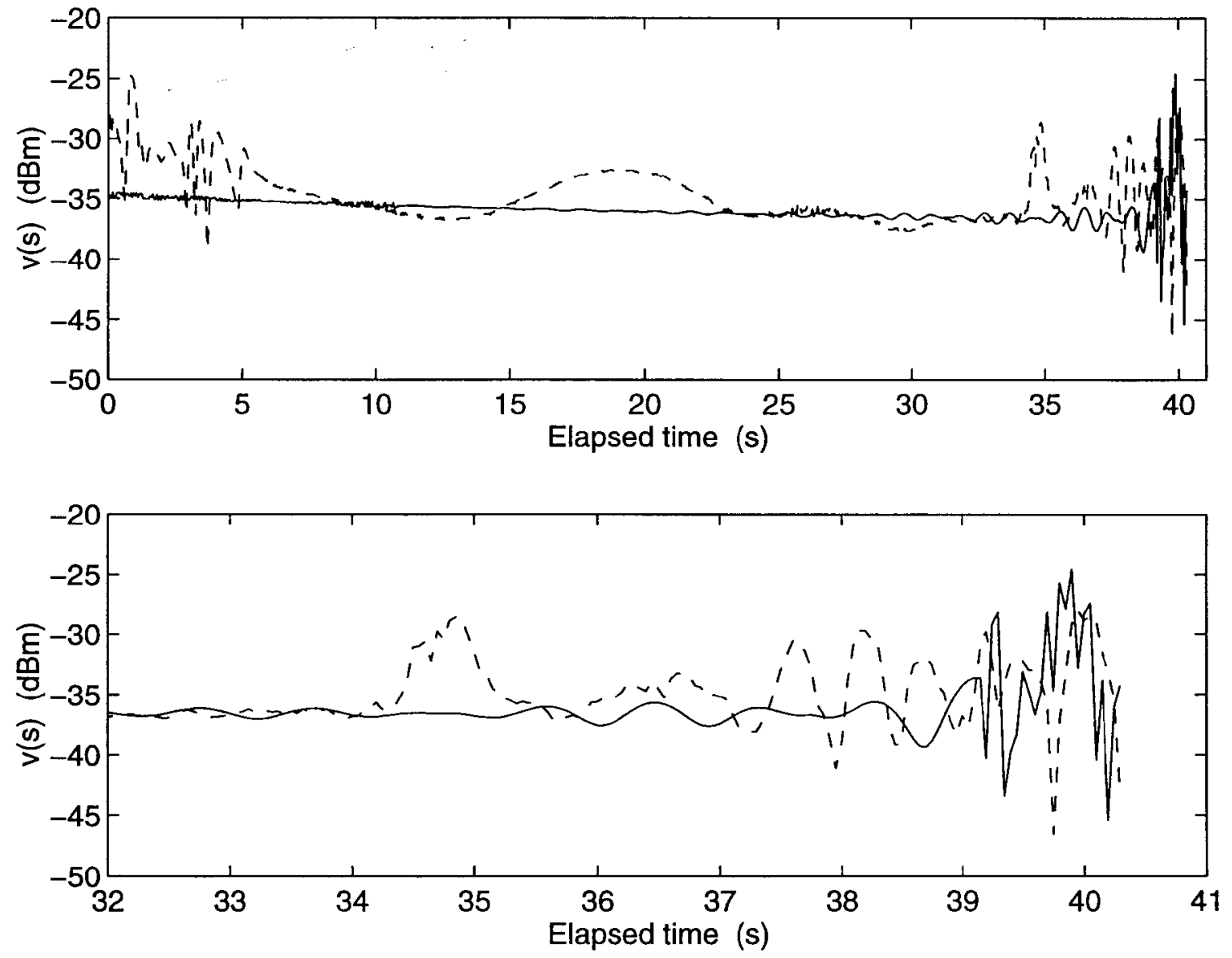

Figure 5: Signal voltage levels from actual data run (dashed line) compared to multipath model taking into account the real land contour, resulting in multiple specular paths (solid line). 